International Journal of Applied Mathematics

Volume 34 No. $3 \quad 2021,441-447$

ISSN: 1311-1728 (printed version); ISSN: 1314-8060 (on-line version)

doi: http://dx.doi.org/10.12732/ijam.v34i3.2

\title{
FIRST ORDER DIFFERENTIAL EQUATION SUDORDINATION ASSOCIATED WITH CASSINI CURVE
}

\author{
Andy Liew Pik Hern ${ }^{1}$, Rashidah Omar ${ }^{2}$, Aini Janteng ${ }^{3} \S$ \\ ${ }^{1,3}$ Faculty of Science and Natural Resources \\ Universiti Malaysia Sabah \\ 88400 Kota Kinabalu, Sabah, MALAYSIA \\ ${ }^{2}$ Faculty of Computer and Mathematical Sciences \\ Universiti Teknologi Mara Cawangan Sabah \\ 88997 Kota Kinabalu, Sabah, MALAYSIA
}

\begin{abstract}
We denote $p(z)$ as analytic functions defined on the open unit disk with $p(0)=1$. In this paper, we determined the condition for $\beta$ so that the results hold for the expressions $1+\beta z p^{\prime}(z), 1+\beta z p^{\prime}(z) / p(z)$ and $1+\beta z p^{\prime}(z) / p^{2}(z)$ are subordinate to $\sqrt{1+c z}$.
\end{abstract}

AMS Subject Classification: 30C45

Key Words: analytic functions; univalent functions; first order differential equation; subordination; Cassini curve

\section{Introduction}

Let $A$ be the class of all the analytic functions of the form

$$
f(z)=z+\sum_{n=2}^{\infty} a_{n} z^{n} \quad(z \in D)
$$

in a unit disk $D=\{z \in \mathbb{C}:|z|<1\}$ and normalized by the condition $f(0)=$ $0=f^{\prime}(0)-1$. We denote $S$ as the subset of $A$ of univalent functions. Also, we denoted $C$ as the class of convex functions and $S^{*}$ as the class of starlike functions. An analytic function $f$ is subordinate to an analytic function $g$, we

Received: June 10, 2020

(C) 2021 Academic Publications

${ }^{\S}$ Correspondence author 
write $f(z) \prec g(z)$ for $z \in D$, if there exists an analytic function $w$ in $D$ such that $w(0)=0$ and $|w(z)|<1$ for $|z|<1$ and $f(z)=g(w(z))$. In particular, if $g$ is univalent in $D$, we say that $f(z) \prec g(z)$ is equivalent to $f(0)=g(0)$ and $f(D) \subset g(D)$.

Goluzin [1] found that if the first order differential subordination $z p^{\prime}(z) \prec$ $z q^{\prime}(z)$ holds and $z q^{\prime}(z)$ is convex, then $p(z) \prec q(z)$ holds where $q$ is the best dominant. Eventually, researchers continued to study about this and the general theory is discussed detailed by Miller and Mocanu in [2]. Nunokawa et al. [3] proved that if $1+z p^{\prime}(z) \prec 1+z$ hold, the subordination $p(z) \prec 1+z$ also hold. There are more results obtained by many other researchers, see [4], [5], [6], [7], [8], [9] and [10].

Sokól and Stankiewicz [11] introduced a class called $S_{L}^{*}$ which consists the function of $f \in A$ such that $w(z):=z f^{\prime}(z) / f(z)$ lies in the region bounded by the right half of the lemniscate of Bernoulli given by $\left|w^{2}-1\right|<1$. This class is associated with the function $\sqrt{1+z}$.

Besides, Aouf et al. [12] defined the class $S^{*}\left(q_{c}\right)$ for $c \in(0,1]$ as:

$$
S^{*}\left(q_{c}\right)=\left\{f \in A:\left|\left[\frac{z f^{\prime}(z)}{f(z)}\right]^{2}-1\right|<c, z \in D\right\} .
$$

It can be established that

$$
f \in S^{*}\left(q_{c}\right) \Leftrightarrow \frac{z f^{\prime}(z)}{f(z)} \prec \sqrt{1+c z} \quad(z \in D) .
$$

We also denoted $\theta_{c}$ as the set of all points in the right half-plane such that the product of the distances from each point to the focuses -1 and 1 is less than $c$ :

$$
\theta_{c}:=\left\{w \in \mathbb{C}: \operatorname{Re} w>0,\left|w^{2}-1\right|<c\right\},
$$

thus the boundary $\partial \theta_{c}$ is the right loop of the Cassinian ovals $\left(x^{2}+y^{2}\right)^{2}-$ $2\left(x^{2}-y^{2}\right)=c^{2}-1$ and for $c=1, S^{*}\left(q_{1}\right) \equiv S_{L}^{*}$.

For an analytic function $p(z)=1+c_{1} z+c_{2} z^{2}+\cdots$, we determine the condition of $\beta$ so that $p(z) \prec P(z)$ where $P(z)$ is a function with positive real part like $\sqrt{1+z}$ and $\varphi_{0}(z):=1+\frac{z}{k}((k+z) /(k-z))(k=\sqrt{2}+1)$, whenever $1+\beta z p^{\prime}(z) / p^{j}(z) \prec \sqrt{1+c z}$, where $j=0,1,2$ (please see [13] for more about $\left.\varphi_{0}(z)\right)$. 


\section{Preliminary results and definitions}

Our results deal with classes of $S^{*}\left(q_{c}\right)$ associated with $S_{L}^{*}$ and $\varphi_{0}(z)$ respectively. Some sufficient conditions with for functions belong to the above defined classes can be obtained by applying the application on starlike functions with positive real part. The first result gives a bound of $\beta$ so that $1+\beta z p^{\prime}(z) \prec \sqrt{1+c z}$ implies that the function $p$ is subordinate to the $\sqrt{1+z}$ function.

Before to get our result, we need the following lemma to prove the theorems.

Lemma 1. ([14]) Let $q$ be analytic in $D$ and let $\psi$ and $v$ be analytic in domain $U$ containing $q(D)$ with $\psi(w) \neq 0$ when $w \in q(D)$. Set $Q(z):=$ $z q^{\prime}(z) \psi(q(z))$ and $h(z):=v(q(z))+Q(z)$. Suppose that:

i. either $h$ is convex or $Q$ is starlike univalent in $D$, and

ii. $\operatorname{Re}\left(z h^{\prime}(z) / Q(z)\right)>0$ for $z \in D$.

If $p$ is analytic in $D$, with $p(0)=q(0), p(D) \subseteq U$ and

$$
v(p(z))+z p^{\prime}(z) \psi(p(z)) \prec v(q(z))+z q^{\prime}(z) \psi(q(z)),
$$

then $p(z) \prec q(z)$ and $q$ is best dominant.

\section{Main results}

Theorem 1. Let the function $p$ be analytic in $D, p(0)=1$ and $1+$ $\beta z p^{\prime}(z) \prec \sqrt{1+c z}, c \in(0,1]$. Then the following subordination results hold:

$$
\text { (a) If } \beta \geq \frac{2[\sqrt{1+c}-\ln (1+\sqrt{1+c})+\ln 2-1]}{\sqrt{2}-1} \text {, }
$$

then $p(z) \prec \sqrt{1+z}$.

$$
\text { (b) If } \beta \geq \frac{2[\sqrt{1-c}-\ln (1-\sqrt{1-c})+\ln 2-1]}{\sqrt{2}-3} \text {, }
$$

then $p(z) \prec \varphi_{0}(z)$.

Proof. The function $q_{B}: \bar{D} \rightarrow \mathbb{C}$ defined by

$$
q_{B}(z)=1+\frac{2}{\beta}[\sqrt{1+c z}-\ln (1+\sqrt{1+c z})+\ln 2-1]
$$


is analytic and it is the solution of $1+\beta z p^{\prime}(z)=\sqrt{1+c z}$. Let $v(w)=1$ and $\psi(w)=\beta$. So the function $Q: \bar{D} \rightarrow \mathbb{C}$ is defined by $Q(z)=z q_{B}^{\prime}(z) \psi\left(q_{B}(z)\right)=$ $\beta z q_{B}^{\prime}(z)$. Since $\sqrt{1+c z}-1$ is starlike function in $D$, it follows that function $Q$ is starlike. Besides, the function $h(z)=v\left(q_{B}(z)\right)+Q(z)$ satisfies $\operatorname{Re}\left(z h^{\prime}(z) / Q(z)\right)>0$ for $z \in D$. Thus, by using Lemma 1 , it shows $1+\beta z p^{\prime}(z) \prec$ $1+\beta z q_{B}^{\prime}(z)$ implies $p(z) \prec q_{B}(z)$. We can say that $p(z) \prec P(z)$ for appropriate $P$ and this holds if the subordination $q_{B}(z) \prec P(z)$ holds. If $q_{B}(z) \prec P(z)$, then $P(-1)<q_{B}(-1)<q_{B}(1)<P(1)$. This gives a necessary condition for $p \prec P$ hold. This necessary condition is sufficient.

(a). By taking $P(z)=\sqrt{1+z}$, the inequalities $q_{B}(-1) \geq P(-1)$ and $q_{B}(1) \leq P(1)$ reduce to $\beta \geq \beta_{1}$ and $\beta \geq \beta_{2}$, where

$$
\beta_{1}=2[\ln (1+\sqrt{1-c})+1-\ln 2-\sqrt{1-c}]
$$

and

$$
\beta_{2}=\frac{2[\sqrt{1+c}-\ln (1+\sqrt{1+c})+\ln 2-1]}{\sqrt{2}-1},
$$

respectively. The subordination $q_{B}(z) \prec \sqrt{1+z}$ holds if $\beta \geq \max \left\{\beta_{1}, \beta_{2}\right\}=\beta_{2}$.

(b). Consider $P(z)=\varphi_{0}(z)$, then the inequalities $q_{B}(-1) \geq \varphi_{0}(-1)$ and $q_{B}(-1) \leq \varphi_{0}(1)$ reduce to $\beta \geq \beta_{1}$ and $\beta \geq \beta_{2}$, where

$$
\beta_{1}=\frac{2[\sqrt{1-c}-\ln (1+\sqrt{1-c})+\ln 2-1]}{2 \sqrt{2}-3}
$$

and

$$
\beta_{2}=2[\sqrt{1+c}-\ln (1+\sqrt{1+c})+\ln 2-1],
$$

respectively. Thus, the subordination $q_{B}(z) \prec \varphi_{0}(z)$ holds if $\beta \geq \max \left\{\beta_{1}, \beta_{2}\right\}=$ $\beta_{1}$.

When $c=1$, we may get Corollary 5 . The next result gives bound on $\beta$ so that $1+\beta z p^{\prime}(z) / p(z) \prec \sqrt{1+c z}$ implies $p$ is subordinate to $\varphi_{0}(z)$ function.

Theorem 2. Let the function $p$ be analytic in $D, p(0)=1$ and $1+$ $\beta z p^{\prime}(z) / p(z) \prec \sqrt{1+c z}, c \in(0,1]$. Then the following subordination result holds:

$$
\text { If } \beta \geq \frac{2[\sqrt{1-c}-\ln (\sqrt{1-c}+1)+\ln 2-1]}{\ln (2 \sqrt{2}-2)} \text {, then } p(z) \prec \varphi_{0}(z) \text {. }
$$


Proof. The function $q_{B}: \bar{D} \rightarrow \mathbb{C}$ defined by

$$
q_{B}(z)=\exp \left\{1+\frac{2}{\beta}[\sqrt{1+c z}-\ln (1+\sqrt{1+c z})+\ln 2-1]\right\}
$$

is analytic and is the solution of $1+\beta z p^{\prime}(z) / p(z)=\sqrt{1+c z}$. Define $v(w)=1$ and $\psi(w)=\beta / w$. The function $Q: \bar{D} \rightarrow \mathbb{C}$ defined by $Q(z):=z q_{B}^{\prime}(z) \psi\left(q_{B}(z)\right)=$ $\beta z q_{B}^{\prime}(z) / q_{B}(z)=\sqrt{1+c z}-1$ is starlike in $D$. The function $h(z):=v\left(q_{B}(z)\right)+$ $Q(z)=1+Q(z)$ satisfies $\operatorname{Re}\left(z h^{\prime}(z) / Q(z)\right)>0$ for $z \in D$. Therefore, by using Lemma 1, we get

$$
1+\beta \frac{z p^{\prime}(z)}{p(z)} \prec 1+\beta \frac{z q_{B}^{\prime}(z)}{q_{B}(z)}
$$

that implies $p(z) \prec q_{B}(z)$. In the similar lines of the proof of Theorem 2, the proof of the result is completed. By substituting $c=1$, we get the result in Corollary 6.

Next, we determine a bound on $\beta$ so that $1+\beta z p^{\prime}(z) / p^{2}(z) \prec \sqrt{1+c z}$ implies $p$ is subordinate to $\varphi_{0}(z)$.

Theorem 3. Let the function $p$ be analytic in $D, p(0)=1$ and $1+$ $\beta z p^{\prime}(z) / p^{2}(z) \prec \sqrt{1+c z}, c \in(0,1]$. Then the following subordination results hold:

$$
\text { If } \beta \geq \frac{4(\sqrt{2}-1)(\sqrt{1-c}-\ln (\sqrt{1-c}+1)+\ln 2-1)}{2 \sqrt{2}-3},
$$

then $p(z) \prec \varphi_{0}(z)$.

Proof. The function $q_{B}: \bar{D} \rightarrow \mathbb{C}$ defined by

$$
q_{B}(z)=\left(1+\frac{2}{\beta}[\sqrt{1+c z}-\ln (1+\sqrt{1+c z})+\ln 2-1]\right)^{-1}
$$

is analytic. It is the solution of $1+\beta z p^{\prime}(z) / p^{2}(z)=\sqrt{1+c z}$. Define $v(w)=1$ and $\psi(w)=\beta / w^{2}$. The function $Q: \bar{D} \rightarrow \mathbb{C}$ defined by

$$
Q(z):=z q_{B}^{\prime}(z) \psi\left(q_{B}(z)\right)=\beta z q_{B}^{\prime}(z) / q_{B}^{2}(z)=\sqrt{1+c z}-1
$$

is starlike in $D$, so $Q$ is starlike function. The function $h(z):=v\left(q_{B}(z)\right)+Q(z)=$ $1+Q(z)$ satisfies $\operatorname{Re}\left(z h^{\prime}(z) / Q(z)\right)>0$ for $z \in D$. Therefore, by using Lemma 1 , we get that

$$
1+\beta \frac{z p^{\prime}(z)}{p^{2}(z)} \prec 1+\beta \frac{z q_{B}^{\prime}(z)}{q_{B}^{2}(z)}
$$


implies $p(z) \prec q_{B}(z)$. As the similar lines of the proof of Theorem 2 the proof of the result is completed.

Also, let $c=1$, we have the result in Corollary 7 .

\section{Corollaries}

Corollary 4. ([10]) Let the function $p$ be analytic in $D, p(0)=1$ and $1+\beta z p^{\prime}(z) \prec \sqrt{1+c z}$. Then the following subordination results hold:

$$
\text { (a) If } \beta \geq \frac{2[\sqrt{2}-1+\ln 2-\ln (1+\sqrt{2})]}{\sqrt{2}-1} \approx 1.09116,
$$

then $p(z) \prec \sqrt{1+z}$.

(b) If $\beta \geq \frac{2(1-\ln 2)}{3-2 \sqrt{2}} \approx 3.57694$, then $p(z) \prec \varphi_{0}(z)$.

Corollary 5. ([10]) Let the function $p$ be analytic in $D, p(0)=1$ and $1+\beta z p^{\prime}(z) / p(z) \prec \sqrt{1+c z}$. Then the following subordination results hold:

$$
\text { If } \beta \geq \frac{2(\ln 2-1)}{\ln (2 \sqrt{2}-2)} \approx 3.26047, \text { then } p(z) \prec \varphi_{0}(z) \text {. }
$$

Corollary 6. ([10]) Let the function $p$ be analytic in $D, p(0)=1$ and $1+\beta z p^{\prime}(z) / p^{2}(z) \prec \sqrt{1+c z}$. Then the following subordination results hold:

$$
\text { If } \beta \geq 4(1+\sqrt{2})(1-\ln 2) \approx 2.96323, \text { then } p(z) \prec \varphi_{0}(z) \text {. }
$$

\section{Acknowledgements}

We thank to UMSGreat Grant, GUG0269-2/2018 for financial support and all the anonymous papers as references.

\section{References}

[1] G.M. Goluzin, On the majorization principle in function theory, Dokl. Akad. Nauk. SSSR, 42 (1935), 647-650. 
[2] S.S. Miller, P.T. Mocanu, On some classes of first-order differential subordinations, Michigan Math. J., 32, No 2 (1985), 185-195.

[3] M. Nunokawa, M. Obradović, S. Owa, On criterion for univalency, Proc. Amer. Math. Soc., 106, No 4 (1989), 1035-1037.

[4] R.M. Ali, V. Ravichandran, N. Seenivasagan, Sufficient conditions for Janowski starlikeness, Int. J. Math. Math. Sci., 2007 (2007), Art. ID 62927, 7 pp.

[5] R. Omar, S.A. Halim, Differential subordinations properties of SokółStankiewicz starlike functions, Kyungpook Math. J. 53, No 3 (2013), 459465.

[6] R. Omar, S.A. Halim, R.W. Ibrahim, Differential subordination properties of certain analytic functions, Int. J. of Math., 21, No 6 (2013), 7 pp.

[7] N.E. Cho, H.J. Lee, J. H. Park, R. Srivastava, Some application of the firstorder differential subordinations, Filomat, 30, No 6 (2016), 1465-1474.

[8] V. Ravichandran, K. Sharma, Sufficient conditions for starlikeness, J. Korean Math. Soc., 52, No 4 (2015), 727-749.

[9] K. Sharma, V. Ravichandran, Applications of subordination theory to starlike functions, Bull. Iranian Math. Soc., 42, No 3 (2016), 761-777.

[10] P. Ahuja, S. Kumar, V. Ravichandran, Application of first order differential subordination for functions with positive real part, Stud. Univ. BabeşBolyai Math., 63 (2018), 303-311.

[11] J. Sokół, J. Stankiewicz, Radius of convexity of some subclasses of strongly starlike functions, Zeszyty Nauk. Politech. Rzeszowskiej Mat., No 19 (1996), 101-105.

[12] M.K. Aouf, J. Dziok, J. Sokół, On a subclass of strongly starlike functions, Appl. Math. Lett., 24 (2011), 27-32.

[13] S. Kumar, V. Ravichandran, A subclass of starlike functions associated with a rational function, Southcast Asian Bull. Math., 40, No 2 (2016), 199-212.

[14] S.S. Miller, P.T. Mocanu, Differential Subordination, Dekker, New York (2000). 
